\title{
A convergence study for the Laguerre expansion in the moment equation method for neoclassical transport in general toroidal plasmas
}

\section{$\operatorname{AUTHOR}(S)$ :}

Nishimura, S.; Sugama, H.; Maassberg, H.; Beidler, C. D.; Murakami, S.; Nakamura, Y.; Hirooka, S.

\section{CITATION:}

Nishimura, S.... [et al]. A convergence study for the Laguerre expansion in the moment equation method for neoclassical transport in general toroidal plasmas. PHYSICS OF PLASMAS 2010, 17(8): 082510.

\section{ISSUE DATE:}

2010-08

\section{URL:}

http://hdl.handle.net/2433/147220

\section{RIGHT:}

Copyright 2010 American Institute of Physics. This article may be downloaded for personal use only. Any other use requires prior permission of the author and the American Institute of Physics. The following article appeared in PHYSICS OF PLASMAS17, 082510 (2010) and may be found at 


\title{
A convergence study for the Laguerre expansion in the moment equation method for neoclassical transport in general toroidal plasmas
}

\author{
S. Nishimura, ${ }^{1, a)}$ H. Sugama, ${ }^{1}$ H. Maaßberg, ${ }^{2}$ C. D. Beidler, ${ }^{2}$ S. Murakami, ${ }^{3}$ Y. Nakamura,${ }^{4}$ \\ and S. Hirooka ${ }^{4}$ \\ ${ }^{1}$ National Institute for Fusion Science, Toki 509-5292, Japan \\ ${ }^{2}$ Max-Planck-Institut für Plasmaphysik, EURATOM Association, Teilinstitut Greifswald, \\ Greifswald D-17491, Germany \\ ${ }^{3}$ Department of Engineering, Kyoto University, Kyoto 606-8501, Japan \\ ${ }^{4}$ Graduate School of Energy Science, Kyoto University, Uji 611-0011, Japan
}

(Received 5 February 2010; accepted 16 July 2010; published online 26 August 2010)

\begin{abstract}
The dependence of neoclassical parallel flow calculations on the maximum order of Laguerre polynomial expansions is investigated in a magnetic configuration of the Large Helical Device [S. Murakami, A. Wakasa, H. Maaßberg, et al., Nucl. Fusion 42, L19 (2002)] using the monoenergetic coefficient database obtained by an international collaboration. On the basis of a previous generalization (the so-called Sugama-Nishimura method [H. Sugama and S. Nishimura, Phys. Plasmas 15, 042502 (2008)]) to an arbitrary order of the expansion, the $13 \mathrm{M}, 21 \mathrm{M}$, and $29 \mathrm{M}$ approximations are compared. In a previous comparison, only the ion distribution function in the banana collisionality regime of single-ion-species plasmas in tokamak configurations was investigated. In this paper, the dependence of the problems including electrons and impurities in the general collisionality regime in an actual nonsymmetric toroidal configuration is reported. In particular, qualities of approximations for the electron distribution function are investigated in detail. () 2010 American Institute of Physics. [doi:10.1063/1.3475792]
\end{abstract}

\section{INTRODUCTION}

In the so-called moment equation approach to the neoclassical transport, ${ }^{1-5}$ the Laguerre (Sonine) polynomial expansions for the Legendre order $l=1$ in distribution functions $f_{a 1}^{(l=1)}$ and the linearized collision terms $C_{a}^{\mathrm{L}}\left(f_{a 1}^{(l=1)}\right)$ are used for handling the energy space. ${ }^{6}$ (Exactly speaking, this technique is sometimes used not only for the order $l=1$ but also for $l=0 .{ }^{1}$ In this study investigating the flux-surface averaged flow moments in $\left\langle B f_{a 1}^{(l=1)}\right\rangle$, however, the treatment of the or$\operatorname{der} l=0$ is out of scope.) One main purpose of the expansions is to convert problems including the field particle portion $C_{a b}\left(\left\langle f_{a \mathrm{M}}\right\rangle, f_{b 1}^{(l=1)}\right)$ in the collision term into linear algebraic equations. Since this portion is an integral operator in the velocity space $(v, \xi)\left[v\right.$ : velocity, $\xi \equiv v_{\|} / v$ : pitch-angle parameter] and causes coupling between particle species, its direct implementation in numerical algorithms for kinetic equations is nearly impossible. Therefore the moment equation approach separates the problem into two parts. ${ }^{1}$ One part is the calculation of the viscosity coefficients for given magnetic field (B) configurations, in which the field particle portion is not essential, and the other part is solving the parallel force balance equations expressed in the algebraic form for the given plasma parameters. Because inclusion of the field particle portion is required especially in calculation of parallel plasma flows and currents, various code developments to include it are now being performed, and it is considered that their benchmarking is also required. ${ }^{7-11}$ However, we should note here that there are other important aspects of this orthogonal expansion corresponding to some physical laws of

${ }^{a)}$ Electronic mail: nishimura.shin@lhd.nifs.ac.jp. the order $l=1,{ }^{12}$ and that this expansion can be extended to arbitrary orders. ${ }^{13}$ Hereafter, the notations in Ref. 14, in which the treatment of general orders of the Laguerre expansion is discussed, are followed. One is that the particle and energy conservations $\nabla \cdot\left(n_{a} \mathbf{u}_{a}\right)=0, \nabla \cdot \mathbf{Q}_{a}=$ energy exchange in the macroscopic MHD equilibrium are included as the orders $j=0,1$ in the expansion of the distribution with the flow moments $u_{\| a j}$,

$$
\begin{gathered}
f_{a 1}^{(l=1)}=\frac{2 v \xi}{v_{\mathrm{T} a}^{2}} \sum_{j=0}^{\infty} u_{\| a j} L_{j}^{(3 / 2)}\left\langle f_{a \mathrm{M}}\right\rangle, \\
u_{\| a j} \equiv \frac{c_{j}}{\left\langle n_{a}\right\rangle} \int v \xi L_{j}^{(3 / 2)} f_{a} \mathrm{~d}^{3} \mathbf{v},
\end{gathered}
$$

and thus these conservation laws are retained even when the higher orders $j \geq 2$ are appropriately truncated. Here, $c_{j} \equiv 3 \cdot 2^{j} j ! /(2 j+3) ! !, \quad L_{j}^{(3 / 2)} \equiv L_{j}^{(3 / 2)}\left(x_{a}^{2}\right), \quad$ and $\quad x_{a}^{2} \equiv m_{a} v^{2} /$ $\left(2\left\langle T_{a}\right\rangle\right) \equiv\left(v / v_{\mathrm{T} a}\right)^{2}$. Analogously the collisional momentum conservation

$$
m_{a} \int \mathbf{v} C_{a b}\left(f_{a}, f_{b}\right) \mathrm{d}^{3} \mathbf{v}+m_{b} \int \mathbf{v} C_{b a}\left(f_{b}, f_{a}\right) \mathrm{d}^{3} \mathbf{v}=0
$$

for arbitrary pair of colliding particle species $a, b$ concerns with only order $j=0$ in the expansion of the collision term with the friction moments $C_{\| a j}$, 


$$
\begin{gathered}
\sum_{b}\left[C_{a b}\left(f_{a 1}^{(l=1)},\left\langle f_{b \mathrm{M}}\right\rangle\right)+C_{a b}\left(\left\langle f_{a \mathrm{M}}\right\rangle, f_{b 1}^{(l=1)}\right)\right] \\
=\frac{2 v \xi}{v_{\mathrm{T} a}^{2}} \sum_{j=0}^{\infty} C_{\| a j} L_{j}^{(3 / 2)}\left\langle f_{a M}\right\rangle, \\
C_{\| a j} \equiv \frac{c_{j}}{\left\langle n_{a}\right\rangle} \int \xi L_{j}^{(3 / 2)} C_{a}^{\mathrm{L}}\left(f_{a 1}^{(l=1)}\right) \mathrm{d}^{3} \mathbf{v} \\
=\frac{c_{j}}{\left\langle n_{a}\right\rangle m_{a}} \sum_{b} \sum_{k=0}^{\infty} l_{j+1, k+1}^{a b} u_{\| b k} .
\end{gathered}
$$

Retaining this conservation is required also for consistency with the macroscopic MHD equilibrium $c \nabla \Sigma p_{a}=\mathbf{J} \times \mathbf{B}$, $\mathbf{J} \equiv \sum e_{a} n_{a} \mathbf{u}_{a}$. Furthermore, truncating higher orders $j \geq 2$ in Eqs. (1) and (3) does not break the self-adjoint property,

$$
\begin{aligned}
& \int \hat{g}_{a} C_{a b}\left[\left\langle f_{a \mathrm{M}}\right\rangle\left(1+\hat{f}_{a}\right),\left\langle f_{b \mathrm{M}}\right\rangle\left(1+\hat{f}_{b}\right)\right] \mathrm{d}^{3} \mathbf{v} \\
& +\int \hat{g}_{b} C_{b a}\left[\left\langle f_{b \mathrm{M}}\right\rangle\left(1+\hat{f}_{b}\right),\left\langle f_{a \mathrm{M}}\right\rangle\left(1+\hat{f}_{a}\right)\right] \mathrm{d}^{3} \mathbf{v} \\
& =\int \hat{f}_{a} C_{a b}\left[\left\langle f_{a \mathrm{M}}\right\rangle\left(1+\hat{g}_{a}\right),\left\langle f_{b \mathrm{M}}\right\rangle\left(1+\hat{g}_{b}\right)\right] \mathrm{d}^{3} \mathbf{v} \\
& \quad+\int \hat{f}_{b} C_{b a}\left[\left\langle f_{b \mathrm{M}}\right\rangle\left(1+\hat{g}_{b}\right),\left\langle f_{a \mathrm{M}}\right\rangle\left(1+\hat{g}_{a}\right)\right] \mathrm{d}^{3} \mathbf{v},
\end{aligned}
$$

for $f_{a}=\left\langle f_{a \mathrm{M}}\right\rangle\left(1+\hat{f}_{a}\right), f_{b}=\left\langle f_{b \mathrm{M}}\right\rangle\left(1+\hat{f}_{b}\right)$ of the arbitrary pair $a, b$. In Eq. (3), this characteristic for the Legendre order $l=1$ is expressed by the symmetric relation of the friction coefficient ${ }^{1} l_{j i}^{b a}=l_{i j}^{a b}$. It is also well known that ${ }^{12}$ the momentum conservation equation (2) results in the intrinsic ambipolarity of the collisional diffusion in the axisymmetric, helically symmetric, and poloidally symmetric conditions of the B-field strength on the flux-surfaces, ${ }^{5}$ and this selfadjoint property results in the Onsager symmetry of the transport matrix. ${ }^{4}$ Confirming these characteristics of the transport coefficients is an important and convenient selfcheck for code developments.

Although many previous studies ${ }^{1-5,7-10}$ were often done with the expansions retaining only two terms $(j=0,1)$ in Eqs. (1) and (3) since it is a minimum choice to include the likeparticle friction collision, these kinds of theories can be extended to include higher orders, as discussed in Ref. 13. Following this methodology, we previously generalized a theory for nonsymmetric toroidal configurations in Ref. 5 (SugamaNishimura method) to include arbitrary Laguerre orders in Eqs. (1) and (3). ${ }^{14}$ However, it also should be noted that requiring much higher orders $j \gg 1$ in calculating $\left\langle B f_{a 1}^{(l=1)}\right\rangle$ is questionable from two viewpoints. One is that it is physically unnatural. This issue is caused by the linearizing approximation neglecting the nonlinear term $C_{a b}\left(f_{a 1}, f_{b 1}\right)$. When the kinetic equations are linearized by this kind of omission, arbitrary modes and/or orders $(m, n, l, j)$ of the orthogonal expansions are allowed mathematically. However, the actual physics is nonlinear. The distribution functions $f_{a}$ should simultaneously satisfy $f_{a}=\left\langle f_{a \mathrm{M}}\right\rangle+f_{a 1}>0$ and $\int v^{2 n} f_{a} \mathrm{~d}^{3} \mathbf{v}$, $\int \mathbf{v} v^{2 n} f_{a} \mathrm{~d}^{3} \mathbf{v}=$ finite. Since the latter characteristic is more important in many practical applications, we use the orthogonally expanded expressions guaranteeing it. However, $f_{a}>0$ is not guaranteed in those kinds of linearized theories and/or codes. When truncating the Laguerre series at an appropriate order $j_{\text {max }}$, inclusion of the higher Laguerre orders $j \gg 1$ prevents the smooth monotonic decrease of $f_{a}$ keeping $f_{a}>0$ at a high energy tail $x_{a} \gg 1$. This is an essential difference between the Fourier-Legendre $(m, n, l)$ expansion for $(\theta, \zeta, \xi)$ space and the Laguerre $(j)$ expansion for the $v$-space. The second problem is that increasing the matrix size often increases numerical errors. For these two reasons, the matrix size should be minimized as effectively as possible, especially when the number of particle species is large. A main conclusion of a previous comparison of so-called $13 \mathrm{M}\left(j \leq j_{\max }=1\right), 21 \mathrm{M}\left(j \leq j_{\max }=2\right)$, and $29 \mathrm{M}\left(j \leq j_{\max }=3\right)$ approximations to answer to this question was that the $13 \mathrm{M}$ approximation is sufficiently accurate for practical purposes. ${ }^{14}$ However, this previous numerical example in Ref. 14 was made (1) only for the ion distribution function in plasmas consisting of a single species of ion, (2) only for banana collisionality regime in tokamak configurations, and (3) by using a small mass ratio approximation to neglect the nondiagonal coupling between electrons and the ions. Therefore, the dependence of the viscosity coefficients on ambipolar radial electric fields, which is peculiar to nonsymmetric stellarator/heliotron configurations, was not included. Furthermore, the Onsager symmetric bootstrap current and Ware pinch coefficients in multi-ion-species plasmas were also not investigated in that study. In this study, the calculation is extended to fully include the nondiagonal coupling between arbitrary particle species including the electrons and impurity ions and the dependence of the flows and the transport coefficients on the $\mathbf{E} \times \mathbf{B}$ effect is investigated.

\section{EXTENSION FOR THE CONVERGENCE STUDY}

Previous studies often show approximated analytical solutions obtained by omitting nondiagonal coupling between particle species. An example is the $13 \mathrm{M}$ approximation for plasmas consisting of only electrons and a single species of ions in Appendix C of Ref. 5, where the ion-electron collision term $C_{\mathrm{ie}}\left(f_{\mathrm{i}}, f_{\mathrm{e}}\right)$ and the inductive parallel electric field $\left\langle B E_{\|}^{(\mathbf{A})}\right\rangle$ in the ion moment equation are treated as the higher order of electron-ion mass ratio. In the present study investigating the bootstrap current and Ware pinch coefficients in multi-ion-species plasmas with an extension to arbitrary Laguerre orders, a method is required to fully include the coupling caused by the field particle portion. Also, the inductive parallel electric field cannot be omitted from the ion moment equations for confirming the Onsager symmetry of the bootstrap current and the Ware pinch. On this Onsager symmetry due to the self-adjoint property, Eq. (4), it was indicated in Ref. 14 that the Laguerre expansion should be performed directly for the kinetic equation. If the kinetic equation is multiplied by inappropriate weighting functions 
in the $v$-space, the intrinsic ambipolarity and the Onsager symmetry are broken. In order to retain momentum conservation and self-adjointness, the following algebraic equation is used, which is obtained by taking the $\int v L_{j}^{(3 / 2)} \mathrm{d}^{3} \mathbf{v}$ Laguerre moments of orders $0 \leq j \leq j_{\max }$ of $\left\langle B \int \xi \mathrm{d} \xi\right\rangle$ of the drift kinetic equation itself. ${ }^{14}$

$$
\left(\left[\begin{array}{ccccc}
\mathbf{M}_{a} & 0 & 0 & \cdots & 0 \\
0 & \mathbf{M}_{b} & 0 & \cdots & 0 \\
0 & 0 & \mathbf{M}_{c} & \cdots & 0 \\
\cdots & \cdots & \cdots & \cdots & \cdots \\
\cdots & \cdots & \cdots & \cdots & \cdots \\
0 & 0 & 0 & \cdots & \mathbf{M}_{N}
\end{array}\right]+\left[\begin{array}{ccccc}
\Lambda^{a a} & \Lambda^{a b} & \Lambda^{a c} & \cdots & \Lambda^{a N} \\
\Lambda^{b a} & \Lambda^{b b} & \Lambda^{b c} & \cdots & \Lambda^{b N} \\
\Lambda^{c a} & \Lambda^{c b} & \Lambda^{c c} & \cdots & \Lambda^{c N} \\
\cdots & \cdots & \cdots & \cdots & \cdots \\
\cdots & \cdots & \cdots & \cdots & \cdots \\
\Lambda^{N a} & \Lambda^{N b} & \Lambda^{N c} & \cdots & \Lambda^{N N}
\end{array}\right]\right)\left[\begin{array}{c}
\mathbf{U}_{a} \\
\mathbf{U}_{b} \\
\mathbf{U}_{c} \\
\cdots \\
\cdots \\
\mathbf{U}_{N}
\end{array}\right]=\left[\begin{array}{ccccc}
\mathbf{N}_{a} & 0 & 0 & \cdots & 0 \\
0 & \mathbf{N}_{b} & 0 & \cdots & 0 \\
0 & 0 & \mathbf{N}_{c} & \cdots & 0 \\
\cdots & \cdots & \cdots & \cdots & \cdots \\
\cdots & \cdots & \cdots & \cdots & \cdots \\
0 & 0 & 0 & 0 & \mathbf{N}_{N}
\end{array}\right]\left[\begin{array}{c}
\mathbf{X}_{a} \\
\mathbf{X}_{b} \\
\mathbf{X}_{c} \\
\cdots \\
\cdots \\
\mathbf{X}_{N}
\end{array}\right]-\left[\begin{array}{c}
\mathbf{Z}_{a} \\
\mathbf{Z}_{b} \\
\mathbf{Z}_{c} \\
\cdots \\
\cdots \\
\mathbf{Z}_{N}
\end{array}\right]\left\langle B E_{\|}^{(\mathbf{A})}\right\rangle .
$$

Here, $\mathbf{M}_{a}$ and $\boldsymbol{\Lambda}^{a b}$ are $\left(j_{\max }+1\right) \times\left(j_{\max }+1\right)$ matrices that include the parallel viscosity coefficients $M_{j+1, k+1}^{a} /\left\langle B^{2}\right\rangle$ expressing the damping force against the flows and the friction coefficients $l_{j+1, k+1}^{a b}$, respectively. The coupling effects between particle species are included by the nondiagonal elements $l_{j+1, k+1}^{a b}(a \neq b)$ corresponding to the field particle portion $C_{a b}\left(\left\langle f_{a \mathrm{M}}\right\rangle, f_{b 1}^{(l=1)}\right)$. The driving forces for the flux-surface-averaged flow moments $\mathbf{U}_{a}$ $=\left[\left\langle B u_{\| a 0}\right\rangle,\left\langle B u_{\| a 1}\right\rangle, \cdots\left\langle B u_{\| a, j_{\max }}\right\rangle\right]^{\text {tr }}$ due to the radial gradient forces $\mathbf{X}_{a}=\left[X_{a 1},-X_{a 2}\right]^{\text {tr }}$ are described by $\mathbf{N}_{a}$, which is the $2 \times\left(j_{\max }+1\right)$ matrix of the nondiagonal viscosity coefficients $N_{j+1, k+1}^{a}$. To include the inductive field $\left\langle B E_{\|}^{(\mathbf{A})}\right\rangle, \mathbf{Z}_{a}$ $=\left[e_{a}\left\langle n_{a}\right\rangle, 0,0, \ldots, 0\right]^{\text {tr }}$, which are vectors with the size of $\left(j_{\max }+1\right)$, are used. The friction coefficients $l_{i j}^{a b}$ for arbitrary Laguerre orders and for arbitrary pair of colliding species $a, b$ can be derived by a method that uses the generating function for Sonine polynomials. ${ }^{6}$ It should also be noted that the higher orders with respect to the electron-ion mass ratio $m_{\mathrm{e}} / m_{\mathrm{i}}$ should be omitted to retain the symmetric relation $l_{j i}^{b a}=l_{i j}^{a b}$ in cases of their different temperatures $T_{\mathrm{e}} \neq T_{\mathrm{i}}$, and also terms of $\left(m_{\mathrm{e}} / m_{\mathrm{i}}\right)^{1 / 2}$ in the ion $(a \neq \mathrm{e})$ friction moment should be retained for the momentum conservation.

By solving this algebraic equation, $\mathbf{U}_{a}$ are given as the linear combination of $\mathbf{X}_{a}$ and $\left\langle B E_{\|}^{(\mathbf{A})}\right\rangle$. We use the direct numerical solution of this equation including the full matrix elements. Thus, the transport coefficients (diffusions, Ware pinch, bootstrap current, and parallel conductivity) are now defined by this numerical solution and cannot be expressed analytically. Though this fact may seem complicated, for computers, it is a simple and straightforward calculation.

\section{VISCOSITY COEFFICIENTS}

As shown in Ref. 14, the viscosity coefficients $M_{j+1, k+1}^{a}$ and $N_{j+1, k+1}^{a}$ in the previous section are obtained by energy integrals of the monoenergetic coefficients $M_{a}(K)$ and $N_{a}(K)$ as functions of $K \equiv x_{a}^{2}$ given by solving the monoenergetic differential equations $\left(V_{\|}+V_{E}-C_{a}^{\mathrm{PAS}}\right) G_{X a}=\sigma_{X a}$ and $\left(V_{\|}+V_{E}\right.$ $\left.-C_{a}^{\mathrm{PAS}}\right) G_{U a}=\sigma_{U a}$ which describe the configuration dependence. Here, $V_{\|} \equiv v_{\|} \mathbf{b} \cdot \nabla_{(\mu=\text { const })}=v \xi \mathbf{b} \cdot \nabla_{(\xi=\text { const })}-(v / 2)$ $\times(\mathbf{b} \cdot \nabla \ln B)\left(1-\xi^{2}\right) \partial / \partial \xi$, where $\mathbf{b}=\mathbf{B} / B$, is the parallel orbit propagator, $V_{E} \equiv c E_{s}\left(\nabla s \times \mathbf{B} /\left\langle B^{2}\right\rangle\right) \cdot \nabla_{(\xi=\text { const })}$ is the $\mathbf{E} \times \mathbf{B}$ operator describing an effect of the ambipolar radial electric field $E_{s} \equiv-\mathrm{d} \Phi / \mathrm{d} s$ on the guiding center orbits [s: arbitrary label of the flux-surfaces], and $C_{a}^{\mathrm{PAS}} \equiv\left(\nu_{\mathrm{D}}^{a} / 2\right)(\partial / \partial \xi)\left(1-\xi^{2}\right)$ $\times(\partial / \partial \xi)$ is the pitch-angle-scattering (PAS) collision operator. The source term $\sigma_{X a}$ in the former equation corresponds to the radial guiding center drift discussed later, and that in the latter equation, which describes the contribution of parallel flows on the viscosity, is defined as $\sigma_{U a} \equiv-m_{a}\left(V_{\|}\right.$ $\left.+V_{E}\right)(v \xi B)$. Since one purpose of this study is the interinstitute benchmarking of the codes having analogous functions, ${ }^{7-11}$ this method to solve the equations should be commonly used ones. Therefore, we basically use the drift kinetic equation solver (DKES) (Ref. 15) monoenergetic coefficient database obtained in an international collaboration for benchmarking these kinds of monoenergetic coefficients. ${ }^{16}$ The assumed magnetic configuration is that with the vacuum axis position of $R_{\mathrm{ax}}=3.6 \mathrm{~m}$ in the large helical device (LHD). ${ }^{17}$

For the parameter $\left(n_{\mathrm{e}}, T_{\mathrm{e}}, T_{\mathrm{i}}, Z_{\mathrm{eff}}\right.$, and $\left.E_{s}\right)$ range scan with multiple-species of ions, however, this energy integral procedure requires a wide range space of the collisionality and the $\mathbf{E} \times \mathbf{B}$ parameter $\left(\nu / v, E_{s} / v\right)$ of the monoenergetic coefficients. This section describes methods to extend the $\left(\nu / v, E_{s} / v\right)$ range for the present study. The function $G_{X a}$ in Refs. 5 and 14 is defined by assuming that the Legendre order $l=0$ of the distribution $f_{a 1}^{(l=0)}$ is determined by the momentum conserving collision operator satisfying Eq. (2) and thus $V_{E} f_{a 1}^{(l=0)}$ is negligibly small. In Ref. 5, the relation between this function and another function $F_{1}$ calculated in the DKES (Ref. 15) was explained by neglecting not only $V_{E} f_{a 1}^{(l=0)}$ but also $V_{E} F_{1}^{(l=0)}$. However, the assumption of $V_{E} F_{1}^{(l=0)} \simeq 0$ is often violated ${ }^{7}$ since an inappropriate collision operator that breaks the momentum conservation determines this function. We analyze this problem first. The equation of $G_{X a}$ given by 


$$
\left(V_{\|}+V_{E}-C_{a}^{\mathrm{PAS}}\right) G_{X a}=\sigma_{X a} \equiv-\sigma_{1}^{+}-\frac{m_{a} c}{e_{a}}\left(V_{\|}+V_{E}\right)(v \xi \tilde{U})
$$

in Refs. 5 and 14 can be rewritten as

$$
\left(V_{\|}+V_{E}-C_{a}^{\mathrm{PAS}}\right)\left(G_{X a}+\frac{m_{a} c}{e_{a}} v \xi \tilde{U}\right)=-\sigma_{1}^{+}+\nu_{\mathrm{D}}^{a} \frac{m_{a} c}{e_{a}} v \xi \tilde{U}
$$

Here, $\quad \sigma_{1}^{+} \equiv-\mathbf{v}_{\mathrm{d} a} \cdot \nabla s \equiv\left(c / e_{a}\right)\left(m_{a} v^{2} / 4\right)\left(1+\xi^{2}\right)(\nabla s \times \mathbf{B}) \cdot \nabla(1 /$ $\left.B^{2}\right)$ is the full component of the radial drift velocity, and $\widetilde{U}$ is the solution of $\mathbf{B} \cdot \nabla(\tilde{U} / B)=(\mathbf{B} \times \nabla s) \cdot \nabla\left(1 / B^{2}\right)$ described in Appendix A of Ref. 5. On the other hand,

$$
\left(V_{\|}+V_{E}-C_{a}^{\mathrm{PAS}}\right) F_{1}=\sigma_{1}^{+},
$$

which is solved in the DKES, ${ }^{15}$ is equivalent to the following equation:

$$
\begin{aligned}
\left(V_{\|}\right. & \left.+V_{E}-C_{a}^{\mathrm{PAS}}\right)\left(F_{1}-\nu_{\mathrm{D}}^{a} \frac{m_{a} c}{e_{a}} \int^{l} \tilde{U} \mathrm{~d} l\right) \\
& =\sigma_{1}^{+}-\nu_{\mathrm{D}}^{a} \frac{m_{a} c}{e_{a}} v \xi \widetilde{U}-\nu_{\mathrm{D}}^{a} \frac{m_{a} c}{e_{a}} V_{E} \int^{l} \tilde{U} \mathrm{~d} l .
\end{aligned}
$$

When the last term in Eq. (9) is negligible, by comparing Eqs. (7) and (9), we obtain

$$
G_{X a}+\frac{m_{a} c}{e_{a}} v \xi \tilde{U} \cong-F_{1}+\nu_{\mathrm{D}}^{a} \frac{m_{a} c}{e_{a}} \int^{l} \tilde{U} \mathrm{~d} l .
$$

Although it is suggested by Eq. (42) in Ref. 5 that we can obtain $G_{X a}$ by solving Eq. (8) (DKES) instead of Eq. (6) with this relation, this substitution is justified only in cases of $\left|\left(e_{a} / c\right) \sigma_{1}^{+}\right| \gg m_{a} \nu_{\mathrm{D}}^{a}\left|V_{E} \int^{l} \tilde{U} \mathrm{~d} l\right|$. The violation of the validity of Eq. (8) in the large $\nu_{\mathrm{D}}^{a} E_{s}$ limit appearing especially for impurity ions in high density and/or high- $Z_{\text {eff }}$ conditions is caused by violation of the momentum conservation. When Eq. (8) is not valid, we cannot use $D_{11}$ and $D_{13}=D_{31}$ defined in Ref. 15 for calculating the neoclassical viscosity effects. The second problem preventing our straightforward energy integral procedure including the large $\nu / v$ limits may be the energy scattering correction to the collision of the Legendre order $l=2$. An essential consideration stated in Refs. 1, 5, and 14 on this correction is that the Pfirsch-Schlüter (PS) energy range, in which $C_{a}^{\mathrm{PAS}} f_{a 1}^{(l=2)}=-3 \nu_{\mathrm{D}}^{a} f_{a 1}^{(l=2)}$ should be replace by the anisotropy relaxation Krook term $-\nu_{\mathrm{T}}^{a} f_{a 1}^{(l=2)}$, is defined by $\nu_{\mathrm{T}}^{a} / v>1 / L_{c}$, where $L_{c}$ is the characteristic length of the $B$-field modulation $1 / L_{c} \simeq \mathbf{b} \cdot \nabla \ln B$. However, this definition is not clear and straightforward in stellarator/heliotron configurations, since two types of modulation coexist: (1) high frequency modulation due to the ripple structure and (2) low frequency modulation due to the toroidicity. A quantitatively clear definition for this situation is $\nu_{\mathrm{T}}^{a} / v>(8 / 5 \pi)$ $\times\left(4 \pi^{2} / V^{\prime}\right)\left|\chi^{\prime} m-\psi^{\prime} n\right| /\left\langle B^{2}\right\rangle^{1 / 2}$ for individual Fourier modes $\sin (m \theta-n \zeta), \cos (m \theta-n \zeta)$ of the distribution and the $B$-field modulation. Here, $\chi^{\prime}, \psi^{\prime}$, and $V^{\prime}$ are radial derivatives $\mathrm{d} / \mathrm{d} s$ of the poloidal flux, toroidal flux, and the volume enclosed by the flux-surface $s=$ const, respectively. In this study requiring the extension to the large $\nu / v$ limit with the finite $E_{s} / v$ values, we shall use the analytical formulas for the plateau and PS energy range shown in Ref. 18. Since these formulas are obtained by solving equations of $G_{X a}$ and $G_{U a}$ directly, the physically meaningless contribution of the $V_{E} F_{1}^{(l=0)}$ is excluded. The dependence of the energy scattering correction on the characteristic length $L_{c}$ is naturally and automatically included by the Fourier expansion. Though the method to include the resonant viscosity effect ${ }^{19}$ is not shown in that study, we can include it by following connection of the monoenergetic analytical formulas in $E_{s} / v$ space. The plateau regime monoenergetic viscosity coefficients that include this effect in the large $E_{s} / v$ limit can be obtained by a method using the aforementioned Krook collision term $-\nu_{\mathrm{T}}^{a}$ and the Fourier expansion. ${ }^{19}$ Though this approximated solution with $E_{s} / v=0$ in collisionless and collisional limits of $\nu / v \rightarrow 0, \infty$ reproduces the exact plateau and PS asymptotic values, as a result of a break of collisional particle and energy conservation $\int \mathrm{d} \xi C_{a}^{\mathrm{L}}\left(G_{X a}\left\langle f_{a \mathrm{M}}\right\rangle\right)=\int \mathrm{d} \xi C_{a}^{\mathrm{L}}\left(G_{U a}\left\langle f_{a \mathrm{M}}\right\rangle\right)=0$, the approximation becomes worth at the transition condition $\nu_{\mathrm{T}}^{a} / v \simeq(8 / 5 \pi)\left(4 \pi^{2} / V^{\prime}\right)\left|\chi^{\prime} m-\psi^{\prime} n\right| /\left\langle B^{2}\right\rangle^{1 / 2}$ of each Fourier modes $(m, n)$. Its use should be limited to the large $E_{s} / v$ range of $\left|b_{m n}\right|>3.0 \times 10^{-2}$, where $b_{m n} \equiv\left(c E_{s} / v\right)$ $\times\left\langle B^{2}\right\rangle^{-1 / 2}\left(B_{\zeta} m+B_{\theta} n\right) /\left(\chi^{\prime} m-\psi^{\prime} n\right)$. Therefore the monoenergetic viscosity coefficients in the plateau and PS energy ranges are given by connecting the function

$$
\left\{\left(\frac{8}{\pi}\left|\chi^{\prime} m-\psi^{\prime} n\right|\right)^{3 / 2}+\left(5 \frac{\nu_{\mathrm{T}}^{a}}{v}\left\langle B^{2}\right\rangle^{1 / 2} \frac{V^{\prime}}{4 \pi^{2}}\right)^{3 / 2}\right\}^{-2 / 3}
$$

in Ref. 18 for $\left|b_{m n}\right|<3.0 \times 10^{-2}$ to the other function expressing the resonance effect,

$$
\begin{aligned}
& \frac{9 a_{m n}}{8\left|\chi^{\prime} m-\psi^{\prime} n\right|} \int_{-1}^{1} \frac{\left(\xi^{2}-1 / 3\right)^{2}}{\left(\xi-b_{m n}\right)^{2}+a_{m n}^{2}} \mathrm{~d} \xi \\
& a_{m n} \equiv \frac{\nu_{\mathrm{T}}^{a}}{v} \frac{\left\langle B^{2}\right\rangle^{1 / 2}}{\left|\chi^{\prime} m-\psi^{\prime} n\right|} \frac{V^{\prime}}{4 \pi^{2}},
\end{aligned}
$$

at $\left|b_{m n}\right|=3.0 \times 10^{-2}$ in the $E_{s} / v$ space. Since this pitch-angle integral can be executed analytically, inclusion of this resonance viscosity effect in the energy integrated coefficients $M_{j+1, k+1}^{a}, N_{j+1, k+1}^{a}$, and $L_{j}^{a}$ does not require a large increase in the computational time.

Figure 1 shows examples of the monoenergetic coefficients (for electrons with $\nu_{\mathrm{T}}^{a} \cong 3 \nu_{\mathrm{D}}^{a}$ ) obtained by the connection in the $v$-space. The open and closed symbols indicate conditions with relatively smaller and larger $\mathbf{E} \times \mathbf{B}$ parameters $E_{r} / v$, respectively. One distinctive characteristic of nonsymmetric heliotron/stellarator configurations can be seen in Fig. 1(b). Compared with symmetric configurations, a deviation from $G^{(\mathrm{BS})}=$ const in the $v$-space may lead to a 

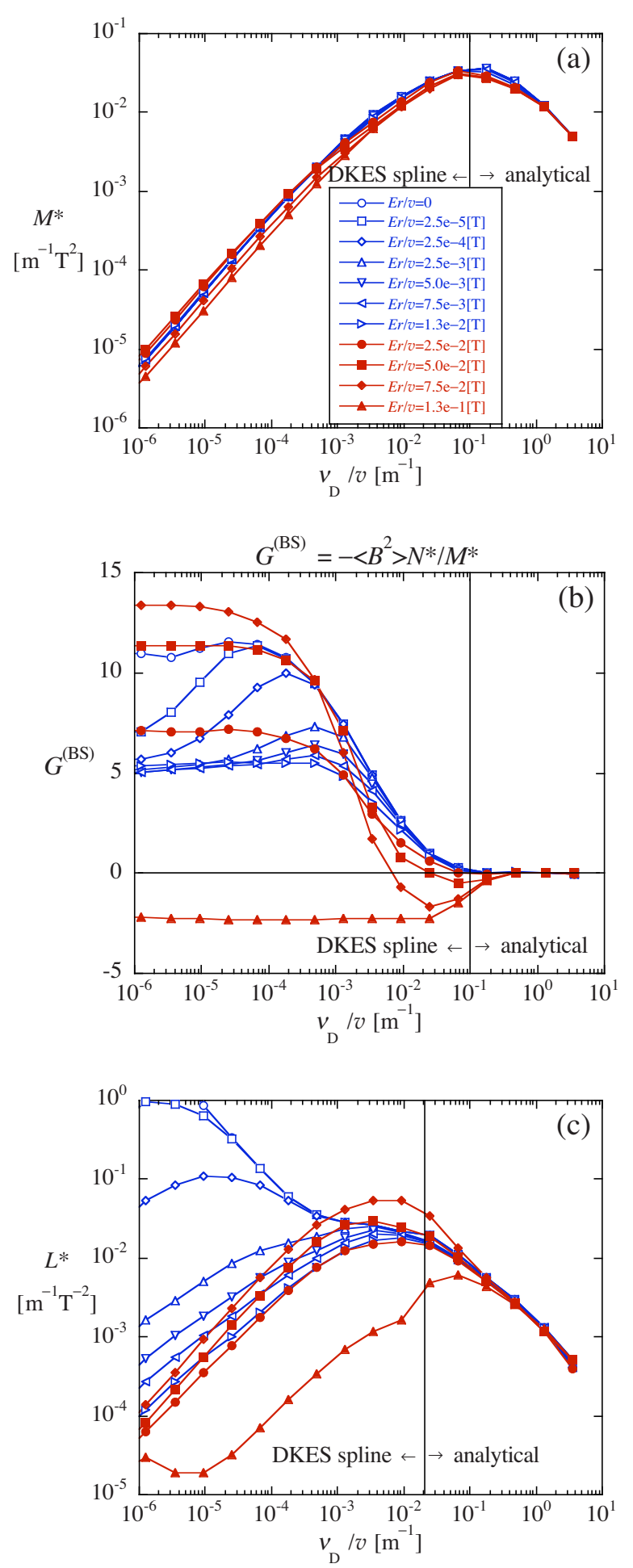

FIG. 1. (Color online) Normalized monoenergetic viscosity coefficients $M^{*}$, $N^{*}$, and $L^{*}$, which are defined in Refs. 5,14 , and 18 , at a radial position of $r / a \cong 0.5$ in the LHD configuration $\left(R_{\mathrm{ax}}=3.6 \mathrm{~m}, B_{00}=2.45 \mathrm{~T}\right)$. Hereafter, the label of the flux-surfaces is the surface averaged minor radius $(s=r[\mathrm{~m}])$ as in these references. The nondiagonal coefficient $N^{*}$ is shown in a normalized form $G^{(\mathrm{BS})} \equiv-\left\langle B^{2}\right\rangle N^{*} / M^{*}$ (so-called geometrical factor) (Refs. 2-5).

complicated fine structure of $\left\langle B f_{a 1}^{(l=1)}\right\rangle$ in the $v$-space, especially when the finite $\mathbf{E} \times \mathbf{B}$ effects are included. One effect is the ripple-trapped/untrapped boundary layer effect, ${ }^{18}$ which is suppressed by a weaker $E_{r} / v$. Another is the resonance viscosity at a large $E_{r} / v$. Even when $c E_{r} / B \ll v_{\mathrm{Ti}}$, which is required for consistency with the stellartor/heliotron equilibrium $^{20}$ with $n_{a} m_{a} \mathbf{u}_{a} \cdot \nabla \mathbf{u}_{a} \ll \nabla p_{a} \Leftrightarrow u_{a} \ll v_{\mathrm{T} a}$, is satisfied, the first toroidal resonant condition $c E_{r} / B \approx v_{\mathrm{Ti}}(\iota / 2 \pi)$ $\times\langle r\rangle /\langle R\rangle$ for the Fourier mode $(m, n)=(1,0)$ often occurs for collisional impurity ions in inner regions in heliotron configurations. ${ }^{21}$ Although we focus on the approximation method for the flux-surface-averaged flow moments in $\left\langle B f_{a 1}^{(l=1)}\right\rangle$ and thus components of radial diffusion fluxes caused by the diagonal coefficient $L^{*}$ are basically out of scope of this paper, also show a connecting result of this coefficient in Fig. 1(c). This result indicates that Eq. (12) can approximate the resonance of the mode $(m, n)=(1,0)$ obtained by the DKES with the pitch-angle-scattering operator that does not break the collisional particle and energy conservation in Eq. (6) at $\nu_{\mathrm{D}}^{a} / v \approx 0.01 \mathrm{~m}^{-1}$.

\section{RESULTS}

The results of the parallel force balance equation (5) with the viscosity coefficients at the radial position $r / a \cong 0.5$ (Fig. 1) are shown in this section. In Figs. 2 and 3, open and closed circles indicate $\left\langle B u_{\| a 0}\right\rangle$ and $\left\langle B u_{\| a 1}\right\rangle$ in Eq. (1), respectively, and other symbols indicate higher order $(j>1)$ moments in the $21 \mathrm{M}$ and $29 \mathrm{M}$ approximations. In Fig. 4, open and closed circles indicate the bootstrap current and Ware pinch coefficients $L_{E 1}^{a}=-L_{1 E}^{a}$ and $-L_{E 2}^{a}=L_{2 E}^{a}$ in the transport matrix, ${ }^{5}$

$$
\begin{aligned}
& {\left[\begin{array}{c}
\left\langle\boldsymbol{\Gamma}_{a}^{\mathrm{bn}} \cdot \nabla s\right\rangle \\
\left\langle\mathbf{q}_{a}^{\mathrm{bn}} \cdot \nabla s\right\rangle /\left\langle T_{a}\right\rangle
\end{array}\right]=\sum_{b}\left[\begin{array}{ll}
L_{11}^{a b} & L_{12}^{a b} \\
L_{21}^{a b} & L_{22}^{a b}
\end{array}\right]\left[\begin{array}{c}
X_{b 1} \\
X_{b 2}
\end{array}\right]+\left[\begin{array}{c}
L_{1 E}^{a} \\
L_{2 E}^{a}
\end{array}\right] X_{E},} \\
& \langle\mathbf{B} \cdot \mathbf{J}\rangle /\left\langle B^{2}\right\rangle^{1 / 2}=\sum_{a}\left[\begin{array}{ll}
L_{E 1}^{a} & L_{E 2}^{a}
\end{array}\right]\left[\begin{array}{l}
X_{a 1} \\
X_{a 2}
\end{array}\right]+\left(L_{E E}+\sigma_{\mathrm{S}}\right) X_{E},
\end{aligned}
$$

with the thermodynamic forces defined by

$$
\begin{aligned}
& X_{a 1} \equiv-\frac{1}{\left\langle n_{a}\right\rangle} \frac{\partial\left\langle p_{a}\right\rangle}{\partial s}-e_{a} \frac{\partial\langle\Phi\rangle}{\partial s}, \quad X_{a 2} \equiv-\frac{\partial\left\langle T_{a}\right\rangle}{\partial s}, \\
& X_{E} \equiv\left\langle B E_{\|}^{(\mathbf{A})}\right\rangle /\left\langle B^{2}\right\rangle^{1 / 2} .
\end{aligned}
$$

In all of these results, thick solid, dot, and thin solid curves indicate the $13 \mathrm{M}, 21 \mathrm{M}$, and $29 \mathrm{M}$ approximations, respectively. As mentioned in the previous sections, these examples take into account two types of energy scattering collisions. One type is that for the Legendre order $l=1$ of the collision term $C_{a}^{\mathrm{L}}\left(f_{a 1}^{(l=1)}\right)$ expressed by the friction coefficient $l_{j+1, k+1}^{a b}$ in Eqs. (3) and (5). It reduces the higher Laguerre order coefficients $\left\langle B u_{\| a j}\right\rangle$ in Eq. (1). The second is that for the order $l=2$ included in the anisotropy relaxation rate $\nu_{\mathrm{T}}^{a}$ in Eqs. (11) and (12), which reduces the higher order coefficients $M_{j+1, k+1}^{a}, N_{j+1, k+1}^{a}$ with $j \geq 2$ in Eq. (5). These effects are large for heavy and high- $Z$ species and are dominated over by the pitch-angle-scattering in collision terms of light and low- $Z$ species. This difference can be seen in the results. Though the result in Fig. 2 for plasmas with single species of the ion with $Z_{\text {eff }}=1$ indicates that the bootstrap current coefficients $L_{E 1}^{a}$ and $L_{E 2}^{a}$, which are determined only by 

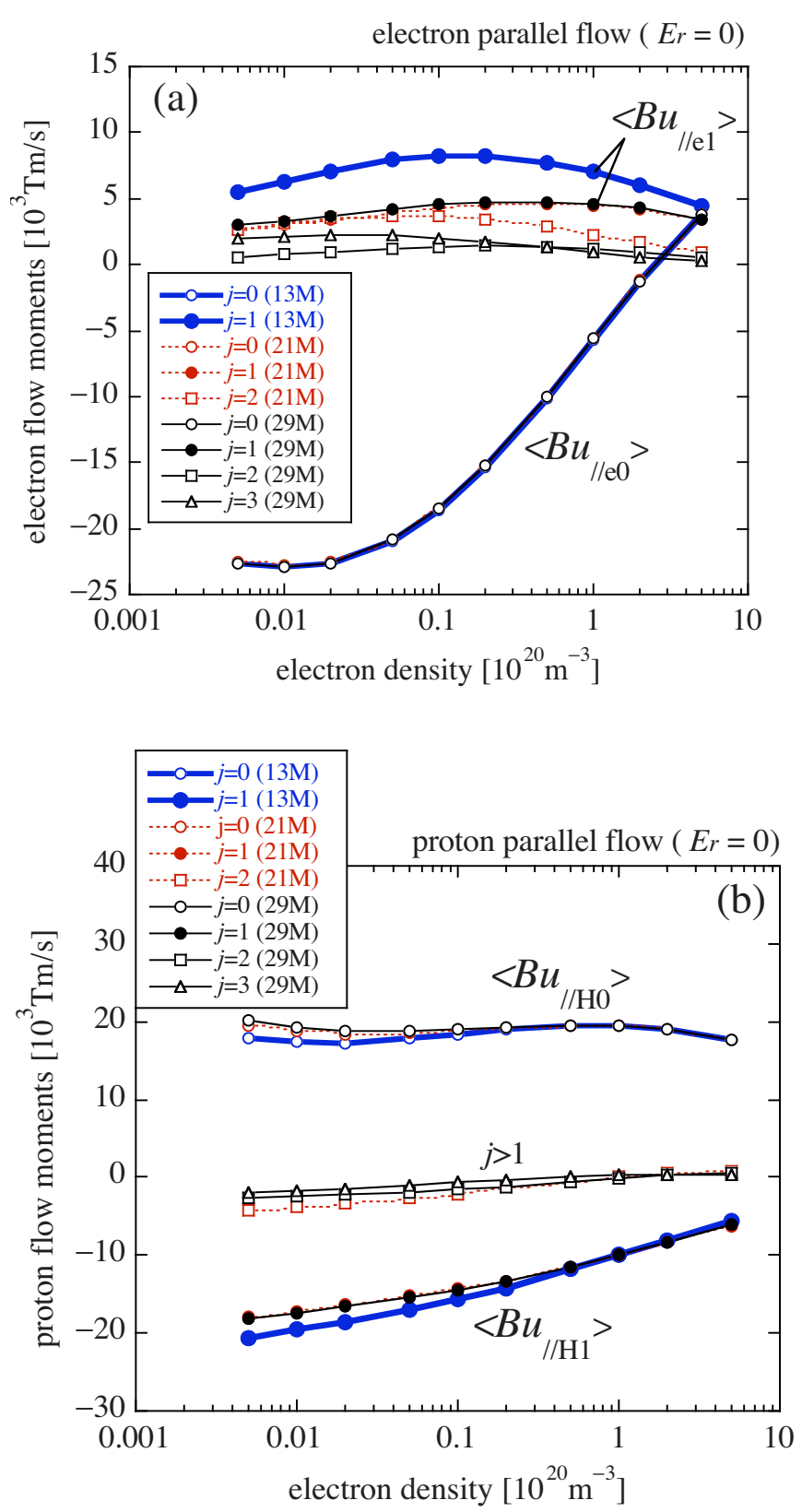

FIG. 2. (Color online) A comparison of flow moments $\left\langle B u_{\| a j}\right\rangle$ for (a) $a=\mathrm{e}^{-}$and (b) $a=\mathrm{H}^{+}$obtained by the $13 \mathrm{M}, 21 \mathrm{M}$, and $29 \mathrm{M}$ approximations as functions of the electron density in $\mathrm{e}^{-}+\mathrm{H}^{+}$plasmas. Assumed parameters are $T_{\mathrm{e}}=T_{\mathrm{i}}=2.0 \mathrm{keV}, \partial p_{\mathrm{e}} / \partial r / n_{\mathrm{e}}=\partial p_{\mathrm{i}} / \partial r / n_{\mathrm{i}}=\partial T_{\mathrm{e}} / \partial r=\partial T_{\mathrm{i}} / \partial r=-3.0 \mathrm{keV} / \mathrm{m}$, and $E_{r}=\left\langle B E_{\|}^{(\mathbf{A})}\right\rangle=0$.

$\left\langle B n_{a} u_{\| a}\right\rangle \equiv\left\langle n_{a}\right\rangle\left\langle B u_{\| a 0}\right\rangle$, can be exactly obtained by the $13 \mathrm{M}$ approximation $\left(j_{\max }=1\right)$; the other result in Fig. 4 indicates that they require the $21 \mathrm{M}$ approximation $\left(j_{\max }=2\right)$ when heavy high- $Z$ impurity ions are included. This requirement is caused by the characteristic of the collision for the light low- $Z$ species. In this situation of the light low- $Z$ species, the structures of their $\left\langle B f_{a 1}^{(l=1)}\right\rangle$ in $v$-space are directly affected by those of their $N_{a}(K), M_{a}(K)$. However, as shown in Figs. 3 and 4 , the $29 \mathrm{M}$ approximation $\left(j_{\max }=3\right)$ is not required for all of these situations even when the complicated $E_{s} / v$ dependence of the boundary layer effect (at $E_{r}<2 \mathrm{kV} / \mathrm{m}$ for $\left.\mathrm{H}^{+}\right)$the resonant viscosity effect $\left(E_{r}>5 \mathrm{kV} / \mathrm{m}\right.$ for $\left.\mathrm{Ne}^{10+}\right)$ are included in $N_{a}(K)$. In these figures, a nonlinear dependence on the radial electric field $E_{r}$ seen in $\left\langle B u_{\| a 0}\right\rangle$ of $\mathrm{Ne}^{10+}$
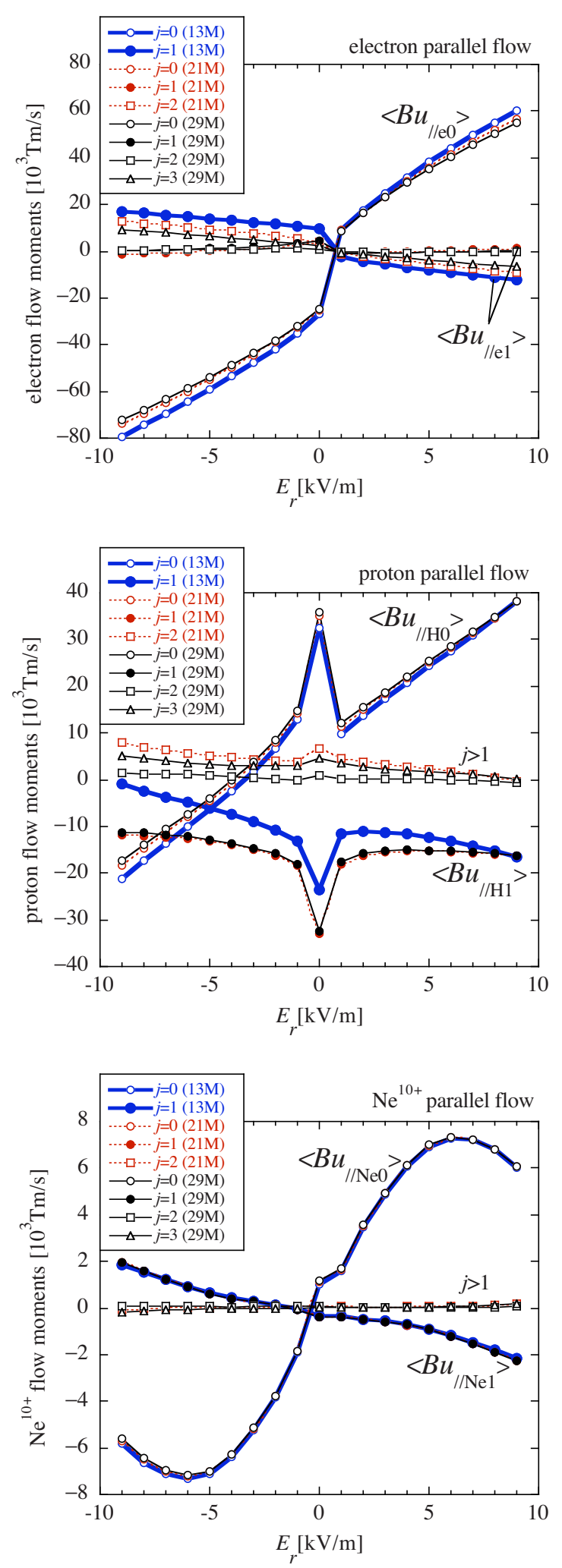

FIG. 3. (Color online) Another comparison of the flow moments $\left\langle B u_{\| a j}\right\rangle$ for $a=\mathrm{e}^{-}, \mathrm{H}^{+}$, and $\mathrm{Ne}^{10+}$ as functions of the radial electric field strength $E_{r}$ by the three approximation methods in $\mathrm{e}^{-}+\mathrm{H}^{+}+\mathrm{Ne}^{10+}$ plasmas. Assumed parameters are $T_{\mathrm{e}}=2.0 \mathrm{keV}, T_{\mathrm{i}}=1.0 \mathrm{keV}, Z_{\text {eff }}=5.74, n_{\mathrm{e}}=1 \times 10^{18} \mathrm{~m}^{-3}$, and $\partial p_{\mathrm{e}} / \partial r / n_{\mathrm{e}}=\partial p_{\mathrm{i}} / \partial r / n_{\mathrm{i}}=\partial T_{\mathrm{e}} / \partial r=\partial T_{\mathrm{i}} / \partial r=-3.0 \mathrm{keV} / \mathrm{m}$.

ions, whose $\nu / v$ value at the thermal velocity corresponds to the plateau regime, indicates the first toroidal resonance. Another fact, which is commonly seen in these dependences on the particle mass, collisionality, and $E_{r}$, is that $\left\langle B f_{a 1}^{(l=1)}\right\rangle$ of the 

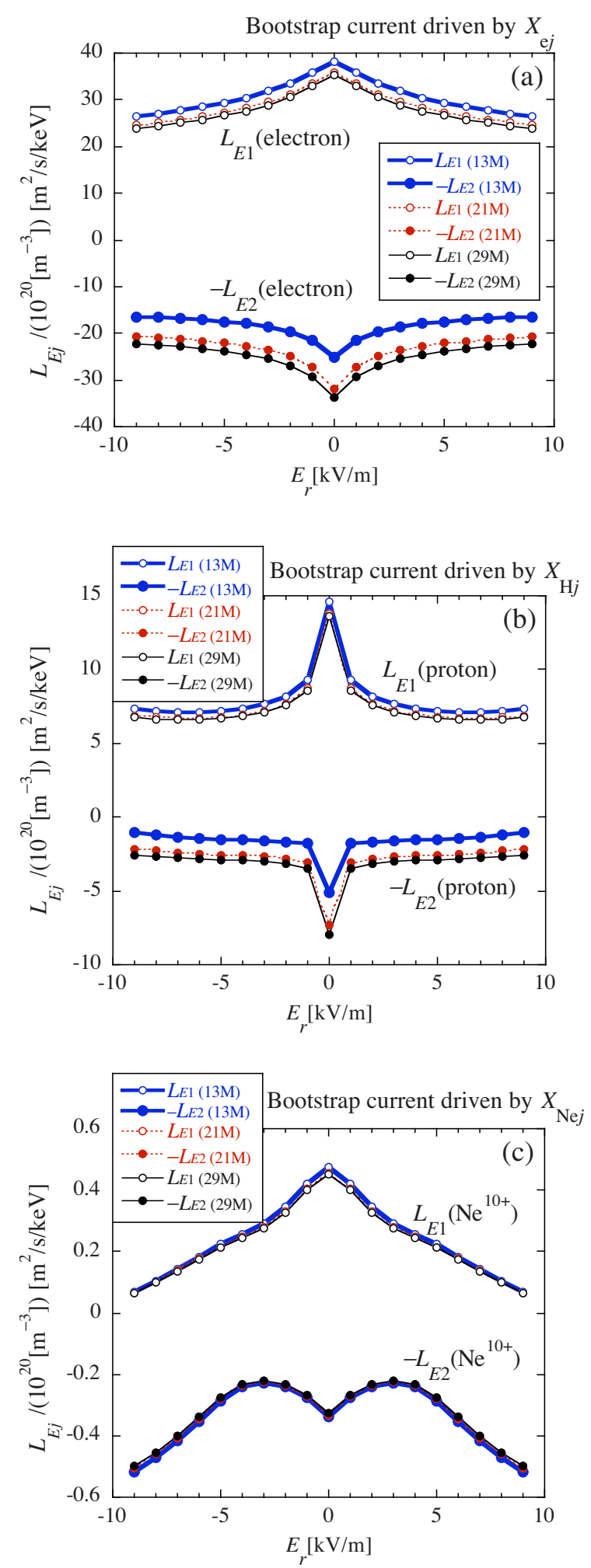

FIG. 4. (Color online) A comparison of the bootstrap current and Ware pinch coefficients $L_{E 1}^{a}=-L_{1 E}^{a}$ and $-L_{E 2}^{a}=L_{2 E}^{a}$ defined in Eq. (13) for the $\mathrm{e}^{-}$ $+\mathrm{H}^{+}+\mathrm{Ne}^{10+}$ plasmas. Figures indicate the bootstrap current driven by (a) the electron forces $a=\mathrm{e}^{-}$, (b) the proton forces $a=\mathrm{H}^{+}$, and (c) the neon forces $a=\mathrm{Ne}^{10+}$. The assumed parameters are those in Fig. 3 .

heaviest particle species is sufficiently approximated by the expansion with only $j_{\max }=1$. This result is due to the energy scattering collision reducing the higher Laguerre order components in $\left\langle B u_{\| a j}\right\rangle, M_{j+1, k+1}^{a}$, and $N_{j+1, k+1}^{a}$ of the heavy species.

\section{CONCLUSION}

The $13 \mathrm{M}\left(j \leq j_{\max }=1\right), 21 \mathrm{M}\left(j \leq j_{\max }=2\right)$, and $29 \mathrm{M}$ $\left(j \leq j_{\max }=3\right)$ approximations for the neoclassical parallel flows are compared in an actual nonaxisymmetric heliotron configuration including the finite radial electric field effects. In contrast with a previous numerical example in Ref. 14, the nondiagonal coupling effects between arbitrary particle species including the electrons are taken into account in this study, and therefore the present results will be useful for future studies on bootstrap current, helium ash control, impurity transport, and so on. The results are summarized as follows.

The flux-surface-averaged component of the distribution $\left\langle B f_{a 1}^{(l=1)}\right\rangle$ of heavy particle species can be approximated by the expansion with only two terms $\left(j_{\max }=1\right)$ in most of practical cases including the situation of the first toroidal resonance of impurities, because of $l=1,2$ energy scattering collisions. The fine structure of $\left\langle B f_{a 1}^{(l=1)}\right\rangle$ in the energy $(v)$ space is smoothed out by this energy scattering effect. Expressing these flux-surface-averaged flow moments of light particle species especially that of electrons sometimes requires the expansion with $j_{\max }=2$. The reason is that $l=1,2$ energy scattering e-e collision effects are small compared with the pitch-angle-scattering e-i collision. In this situation, the structure of $\left\langle B f_{\mathrm{e} 1}^{(l=1)}\right\rangle$ in the $v$-space is directly affected by that of $N_{\mathrm{e}}(K)$ and $M_{\mathrm{e}}(K)$. In impure plasmas, $j_{\max }=2$ is favorable for proton distribution function by the same reason. It should be emphasized here that $j_{\max }=3$ is not required for all of these cases. Though we focused on the parallel flow moments driven by the radial gradient forces in this paper, this calculation also clarified that the parallel conductivity $L_{E E}+\sigma_{\mathrm{S}}$ also is insensitive to this expansion method. Note also that this conclusion on the impurities is for the fluxsurface-averaged parallel flow moments in $\left\langle B f_{a 1}^{(l=1)}\right\rangle$. For the poloidal and toroidal variations $f_{a 1}^{(l=1)}-\left\langle B f_{a 1}^{(l=1)}\right\rangle B /\left\langle B^{2}\right\rangle$ of collisional high- $Z$ species, $j_{\max }=2$ is required to include the $l$ $=0$ energy scattering ion-ion collision. ${ }^{1}$

\section{ACKNOWLEDGMENTS}

The authors acknowledge stimulating discussions with Dr. D. A. Spong, Dr. J. Lore, Dr. M. Yokoyama, and Dr. A. Matsuyama. One author (S.N.) also thanks Professor F. Sano, Professor M. Kikuchi, and Professor K. Hanatani for their encouragement in his collaboration with Kyoto University.

This work is supported by coordinated research programs NIFS06KUHL007 and NIFS07KNXN103 in the National Institute for Fusion Science.

${ }^{1}$ S. P. Hirshman and D. J. Sigmar, Nucl. Fusion 21, 1079 (1981).

${ }^{2}$ K. C. Shaing and J. D. Callen, Phys. Fluids 26, 3315 (1983).

${ }^{3}$ N. Nakajima and M. Okamoto, J. Phys. Soc. Jpn. 61, 833 (1992).

${ }^{4}$ H. Sugama and W. Horton, Phys. Plasmas 3, 304 (1996).

${ }^{5}$ H. Sugama and S. Nishimura, Phys. Plasmas 9, 4637 (2002).

${ }^{6}$ P. Helander and D. J. Sigmar, Collisional Transport in Magnetized Plasmas (Cambridge University Press, New York, 2002), Chap. 4.

${ }^{7}$ D. A. Spong, Phys. Plasmas 12, 056114 (2005).

${ }^{8}$ A. S. Ware, D. A. Spong, L. A. Berry, S. P. Hirshman, and J. F. Lyon, Fusion Sci. Technol. 50, 236 (2006). 
${ }^{9}$ A. Matsuyama, M. Yu. Isaev, K. Y. Watanabe, K. Hanatani, Y. Suzuki, N. Nakajima, W. A. Cooper, and T. M. Tran, Phys. Plasmas 16, 052501 (2009).

${ }^{10}$ J. Lore, W. Guttenfelder, A. Briesemeister, D. T. Anderson, F. S. B. Anderson, C. B. Deng, K. M. Likin, D. A. Spong, J. N. Talmadge, and K. Zhai, Phys. Plasmas 17, 056101 (2010).

${ }^{11}$ H. Maaßberg, C. D. Beidler, and Y. Turkin, Phys. Plasmas 16, 072504 (2009).

${ }^{12}$ R. D. Hazeltine and J. D. Meiss, Plasma Confinement (Addison-Wesley, Redwood City, CA, 1992), Chap. 5.

${ }^{13}$ R. Balescu, Transport Processes in Plasmas (North-Holland, Amsterdam, 1988).

${ }^{14}$ H. Sugama and S. Nishimura, Phys. Plasmas 15, 042502 (2008).

${ }^{15}$ W. I. van Rij and S. P. Hirshman, Phys. Fluids B 1, 563 (1989).
${ }^{16}$ C. D. Beidler, M. Yu. Isaev, S. V. Kasilov, W. Kernbichler, H. Maassberg, D. R. Mikkelsen, S. Murakami, V. V. Nemov, M. Schmidt, D. A. Spong, V. Tribaldos, and A. Wakasa, Proceedings of the 22nd IAEA Fusion Energy Conference, Geneva, Switzerland, 13-18 October 2008 (IAEA, Vienna, 2009), Paper No. TH/P8-10.

${ }^{17}$ S. Murakami, A. Wakasa, C. D. Beidler, H. Maaßberg, H. Yamada, and K. Y. Watanabe, LHD Experimental Group, Nucl. Fusion 42, L19 (2002).

${ }^{18}$ S. Nishimura, H. Sugama, and Y. Nakamura, Fusion Sci. Technol. 51, 61 (2007).

${ }^{19}$ K. C. Shaing, Phys. Fluids B 5, 3841 (1993).

${ }^{20}$ M. Wakatani, Stellarator and Heliotron Devices (Oxford University Press, New York, 1998), Chap. 4.

${ }^{21}$ S. Nishimura, H. Sugama, and CHS Group, Fusion Sci. Technol. 46, 77 (2004). 